\title{
Infección asociada a catéter por Leclercia adecarboxylata y Raoultella ornithinolytica en un paciente con enfermedad mitocondrial

\author{
Leclercia adecarboxylata and Raoultella ornithinolytica catheter-related \\ infection in a child with mitocondrial disease
}

\author{
Lda. María Sánchez-Códeza , Grad. Manuel Lubián-Gutiérrez ${ }^{b}$ Ldo. José A. Blanca-Garcíac y \\ Lda. Cristina Pérez Aragón ${ }^{c}$
}

\begin{abstract}
RESUMEN
Leclercia adecarboxylata y Raoultella ornithinolytica constituyen bacterias Gram-negativas emergentes. Los casos descritos son excepcionales. En los últimos años, las mejoras en las técnicas de diagnóstico microbiológico han permitido su detección y conocimiento. Se presenta el caso de un niño de 11 años con enfermedad mitocondrial, portador de catéter venoso central de larga duración, que desarrolló dos episodios de sepsis por $L$. adecarboxylata y R. ornithinolytica, respectivamente. En los casos de infección asociada al uso de catéter, es posible, en ocasiones, el tratamiento sin su retirada con evolución favorable.

Es importante reconocer L. adecarboxylata y R. ornithinolytica como patógenos de diagnóstico cada vez más frecuentes, sobre todo, en pacientes inmunodeprimidos o con patologías crónicas asociadas.

Palabras clave: sepsis, Enterobacteriaceae, infecciones relacionadas con catéteres, enfermedades mitocondriales, pediatría.
\end{abstract}

\begin{abstract}
Leclercia adecarboxylata and Raoultella ornithinolytica are emergent Gram-negative bacteria. Infections caused by these microorganisms are exceptional. Improvement of microbiologist techniques in the last years has enabled their detection and more accurate knowledge. We present the case of an 11-year-old boy with mitochondrial disease with a longterm central catheter who suffered from two sepsis caused by $L$. adecarboxylata and $R$. ornithinolytica, respectively. In catheter-related infections, sometimes it is possible to provide antimicrobial treatment without removal of catheter with good results, as in our patient.

It is important to recognize L. adecarboxylata and R. ornithinolytica like increasingly frequent pathogenic bacteria, mostly in immunocompromised or chronic patients.

Key words: sepsis, Enterobacteriaceae, catheter-related infections,
\end{abstract} mitochondrial diseases, pediatrics.

a. Departamento de Infectología Pediátrica, Hospital Universitario Puerta del Mar, Cádiz, España.

b. Servicio dePediatría, Hospital Universitario Puerta del Mar, Cádiz, España.

c. Departamento de Gastroenterología y Nutrición de Pediatría, Hospital Universitario Puerta del Mar, Cádiz, España.

Correspondencia:

Lda. María Sánchez Códez: mscodez1990@gmail.com

Financiamiento: Ninguno.

Conflicto de intereses: Ninguno que declarar.

Recibido: $10-4-2018$

Aceptado: 25-10-2018 http: / / dx.doi.org/10.5546/aap.2019.e147

Cómo citar: Sánchez-Códez M, Lubián-Gutiérrez M, Blanca-García JA, Pérez Aragón C. Infección asociada a catéter por Leclercia adecarboxylata y Raoultella ornithinolytica en un paciente con enfermedad mitocondrial. Arch Argent Pediatr 2019;117(2):e147-e149.

\section{INTRODUCCIÓN}

Leclercia adecarboxylata, única especie del género Leclercia, es un bacilo Gram-negativo, móvil y aerobio. ${ }^{1}$ Es ubicuo en la naturaleza; está presente en las aguas, en los líquidos orgánicos y en el tracto gastrointestinal tanto humano como animal. ${ }^{2,3}$ Se ha descrito como agente productor de bacteriemia, peritonitis, ${ }^{4}$ infecciones de piel y tejidos blandos (IPTB) ${ }_{1}^{4}$ neumonía, endocarditis, ${ }^{2}$ absceso periamigdalino ${ }^{5}$ y colecistitis, entre otros.

Raoultella ornithinolytica es otro bacilo Gramnegativo, anaerobio facultativo, procedente del género Klebsiella. Su principal característica es la producción de ácido dicarboxílico. Se presenta como comensal en ambientes acuáticos, aunque también se ha aislado en saliva humana. ${ }^{6}$ Es causante de bacteriemia, infección del tracto urinario (ITU), IPTB, meningitis, mediastinitis, pericarditis, osteomielitis, infecciones del tracto biliar, ${ }^{6}$ conjuntivitis e incluso otitis. ${ }^{7}$

Las infecciones por dichos gérmenes son muy infrecuentes tanto en la población general como en pediatría. ${ }^{4}$ Se presenta nuestra experiencia con estos microorganismos de la familia Enterobacteriaceae en un paciente con enfermedad mitocondrial.

\section{CASO CLÍNICO}

Niño de 11 años con diagnóstico de encefalomiopatía neurogastrointestinal mitocondrial (mitochondrial neurogastrointestinal encephalopathy; MNGIE, por sus siglas en inglés) y déficit de todos los complejos de la cadena respiratoria. Presentaba síndrome de LennoxGastaut en tratamiento con levetiracetam, 
pseudoobstrucción intestinal y malnutrición crónica moderada-grave. Como antecedentes quirúrgicos, se destacaban la realización de funduplicatura laparoscópica de Nissen y era portador de gastrostomía desde los 9 años de edad y catéter de larga duración (Port-a-Cath ${ }^{\circledR}$ ) desde los 10 años.

Ingresó en nuestro Centro por presentar un cuadro de distensión abdominal de dos semanas de evolución, a pesar de las medidas dietéticas y el tratamiento descontaminante con ciclos de metronizadol y trimetoprim-sulfametoxazol. Presentaba mala tolerancia de la nutrición enteral, por lo que precisó, a los quince días de su ingreso, nutrición mixta (enteral y parenteral -NP-).

Al mes de su ingreso, presentó fiebre de hasta $39,5^{\circ} \mathrm{C}$, taquipnea y taquicardia, con hallazgos analíticos de anemia (hemoglobina: 9,1 g/dl), leucopenia $(3480 / \mu 1)$ y reacción en cadena de la polimerasa (polymerase chain reaction; PCR, por sus siglas en inglés) de $100 \mathrm{mg} / 1$. Se extrajeron cultivos de sangre periférica y acceso central, y se suspendió la administración de NP a través de él. Se inició el tratamiento empírico de sepsis con vancomicina intravenosa y sellado del dispositivo Port-a-Cath ${ }^{\circledR}$. Se aisló L. adecarboxylata y Candida parapsilosis en ambas muestras, por lo que se trató de forma dirigida según el antibiograma con amikacina, cefotaxima y fluconazol. Además, se realizó el sellado del catéter y el tratamiento local con amikacina. La duración total del tratamiento dirigido fue de dieciocho días y se reinició, posteriormente, la NP por dispositivo central.

Tras tres colonizaciones del catéter venoso central por gérmenes Gram-positivos (sin desarrollar cuadro de sepsis), se realizó la sustitución del sistema Port-a-Cath ${ }^{\circledR}$ por dispositivo canalizado Hickman monoluz a los 5 meses de su ingreso.

A los seis meses de hospitalización, presentó un segundo episodio de sepsis con fiebre de hasta $40^{\circ} \mathrm{C}$, taquipnea, taquicardia, alteración del nivel de conciencia respecto al estado habitual, anemia (hemoglobina: $9 \mathrm{~g} / \mathrm{dl}$ ) y leucopenia $(3900 / \mu \mathrm{l})$. Se extrajo un hemocultivo de sangre periférica y acceso central, y se inició el tratamiento empírico intravenoso y el sellado del catéter con amikacina. Se aisló $R$. ornithinolytica y Serratia ureilytica de forma exclusiva en el hemocultivo de sangre periférica. El antibiograma mostró sensibilidad a amikacina y el paciente evolucionó de forma favorable tras catorce días de tratamiento. Fue dado de alta con seguimiento en consultas externas de Infectología, Gastroenterología y Neurología Pediátricas. Hasta la fecha, no ha presentado nuevos episodios de infección asociada al catéter, y se mantiene el acceso central de larga duración.

\section{DISCUSIÓN}

L. adecarboxylata se ha descrito, en los últimos años, como patógeno emergente. Se ha asociado con brotes nosocomiales de artritis y bacteriemia en pacientes inmunocompetentes, aunque esta situación es rara. ${ }^{2,4,5}$ Afecta, generalmente, a sujetos con cierto grado de inmunosupresión como agente oportunista y en el contexto de infecciones polimicrobianas, ${ }^{8}$ al igual que en nuestro paciente, con coinfección con C. parasilopsis. Del mismo modo, las infecciones por R. ornithinolytica se han descrito en diabéticos, pacientes oncológicos ${ }^{9,10}$ e inmunodeprimidos. ${ }^{6}$

No está claro el mecanismo por el que L. adecarboxylata alcanza la circulación. Se han comunicado varios casos de infección tras heridas con solución de continuidad en la barrera cutánea (traumatismos y quemaduras). Igualmente, se han expuesto bacteriemias en pacientes con patología digestiva, como úlcera péptica, ${ }^{11}$ colecistitis y colitis ulcerosa, debido a alteraciones de la flora saprófita y aumento de permeabilidad gastrointestinal. ${ }^{3} \mathrm{Se}$ han descrito bacteriemias asociadas al uso de catéter, sobre todo, de diálisis peritoneal. Más infrecuentes son las relacionadas con el catéter vascular (especialmente, centrales, permanentes y tunelizados) para NP, como nuestro caso, o quimioterapia. ${ }^{12}$ De $R$. ornithinolytica se destaca su capacidad para adherirse a las superficies tisulares y formar biofilms, lo que explica las infecciones relacionadas con el uso de catéteres urinarios, venosos y dispositivos ortopédicos. ${ }^{7}$ En nuestro caso, los factores de riesgo para el desarrollo de sepsis son variados: portador del catéter venoso central permanente, alteración de la microbiota intestinal y translocación bacteriana secundaria a patología de base.

Ambas enterobacterias se destacan por su abordaje terapéutico sencillo, ${ }^{11,13}$ gracias a su susceptibilidad antibiótica. Se han descrito casos aislados de multirresistencia, que es uno de los factores que más contribuyen a su mortalidad. ${ }^{9}$ De los dos microorganismos, la mayoría de las especies resistentes descritas se refieren a Leclercia ${ }^{14}$ productora de beta-lactamasas. Esta última es sensible a todos los antimicrobianos efectivos contra Enterobacteriaceae, salvo fosfomicina, a la cual presenta resistencia natural 
(aspecto característico y que puede ayudar a distinguir Leclercia de E. coli). ${ }^{13}$ Además, se han descrito algunas especies de Raoultella productoras de beta-lactamasas que confieren resistencia a carbapenem, antibiótico de elección en las infecciones graves nosocomiales. ${ }^{15}$

Nuestro paciente, como la mayoría de los casos revisados, presentó excelente respuesta terapéutica a los antibióticos de elección. Existen casos, como este, en los que ha sido posible el tratamiento de la infección asociada al catéter sin retirar el dispositivo, llevando a cabo el sellado del catéter con ciprofloxacino o gentamicina. ${ }^{12}$

Como conclusión, se destaca el papel excepcional de las infecciones por $L$. adecarboxylata y R. ornithinolytica. Afectan, sobre todo, a pacientes inmunodeprimidos o con patología de base, en especial, portadores de dispositivos, como el catéter venoso central. Se destaca la evolución favorable en nuestro caso de la infección asociada al catéter tratada con antimicrobianos exclusivamente.

\section{REFERENCIAS}

1. Atas DB, Velioglu A, Asicioglu E, Arikan H, et al. Polymicrobial peritonitis with Leclercia adecarboxylatain a peritoneal dialysis patient. Saudi J Kidney Dis Transpl. 2017; 28(1):181-2.

2. Keren $Y$, Keshet D, Eidelman M, Geffen Y, et al. Is Leclercia adecarboxylata a new and unfamiliar marine pathogen? J Clin Microbiol. 2014; 52(5):1775-6.

3. Kashani A, Chitsazan M, Che K, Garrison RC. Leclercia adecarboxylata Bacteremia in a Patient with Ulcerative Colitis. Case Rep Gastrointest Med. 2014; 2014:457687.

4. Grantham WJ, Funk SS, Schoenecker JG. Leclercia adecarboxylata Musculoskeletal Infection in an Immune
Competent Pediatric Patient: An Emerging Pathogen? Case Rep Orthop. 2015; 2015:160473.

5. Bali R, Sharma P, Gupta K, Nagrath S. Pharyngeal and peritonsillar abscess due to Leclercia adecarboxylata in an immunocompetant patient. J Infect Dev Ctries. 2013;7(1):4650.

6. Seng P, Boushab BM, Romain F, Gouriet F, et al. Emerging role of Raoultella ornithinolytica in human infections: a series of cases and review of the literature. Int J Infect Dis. 2016; 45:65-71.

7. Seng P, Theron F, Honnorat E, Prost D, et al. Raoultella ornithinolytica: An unusual pathogen for prosthetic joint infection. IDCases. 2016; 5:46-8.

8. Anuradha M. Leclercia adecarboxylata isolation: case reports and review. J Clin Diagn Res. 2014; 8(12):DD03-4.

9. Kaya S, Bayramoğlu G, Sönmez M, Köksal İ. Raoultella ornithinolytica causing fatal sepsis. Braz J Infect Dis. 2015; 19(2):230-1.

10. Hadano Y, Tsukahara M, Ito K, Suzuki J, et al. Raoultella ornithinolytica bacteremia in cancer patients: report of three cases. Intern Med. 2012; 51(22):3193-5.

11. JeanSS, LeeWS, BaiKJ, Lam C, etal. Leclercia adecarboxylata bacteremia in a patient with long-term use of nonsteroidal anti-inflammatory drugs. J Microbiol Immunol Infect. 2016; 49(3):452-4

12. Fernández-Ruiz M, López-Medrano F, García-Sánchez L, García-Reyne A, et al. Successful management of tunneled hemodialysis catheter-related bacteremia by Leclercia adecarboxylata without catheter removal: report of two cases. Int J Infect Dis. 2009; 13(6):e517-8.

13. Stock I, Burak S, Wiedemann B. Natural antimicrobial susceptibility patterns and biochemical profiles of Leclercia adecarboxylata strains. Clin Microbiol Infect. 2004;10(8):72433.

14. Mazzariol A, Zuliani J, Fontana R, Cornaglia G. Isolation from blood culture of a Leclercia adecarboxylata strain producing an SHV-12 extended-spectrum beta-lactamase. J Clin Microbiol. 2003; 41(4):1738-9.

15. Khajuria A, Praharaj AK, Grover N, Kumar M. First report of blaNDM-1 in Raoultella ornithinolytica. Antimicrob Agents Chemother. 2013; 57(2):1092-3. 